\begin{tabular}{|c|l|}
\hline Title & Moth sex chromatin probed by comparative genomic hybridization (CGH) \\
\hline Author(s) & Sahara, Ken; Marec, FrantiDek; Eickhoff, Ulrike; Traut, Walther \\
\hline Citation & Genome, 46(2), 339-342 \\
\hline Issue Date & 2003-04 \\
\hline Doc URL & http://hdl.handle.net/2115/891 \\
\hline Rights & Copyright (c) 2003 National Research Council of Canada \\
\hline Type & article \\
\hline File Information & Genome46(2).pdf \\
\hline
\end{tabular}

Instructions for use 
Genome 46, 339-342, 2003. post-reviewed MS

\title{
NOTE
}

\section{Moth sex chromatin probed by comparative genomic hybridization} (CGH)

\author{
Ken Sahara ${ }^{1)}$, František Marec ${ }^{2)}$, Ulrike Eickhoff ${ }^{3)} \quad \&$ Walther Traut ${ }^{3)}$
}

1) Division of Applied Biosciences, Graduate School of Agriculture, Hokkaido University, Sapporo 060-8589, Japan, sahara@abs.agr.hokudai.ac.jp

2) Institute of Entomology, Czech Academy of Sciences, CZ-37005 České Budějovice, Czech Republic, marec@entu.cas.cz

3) Institut für Biologie, Medizinische Universität zu Lübeck, D-23538 Lübeck, Germany, traut@molbio.mu-luebeck.de

running title: Moth sex chromatin probed by $\mathrm{CGH}$

Corresponding author:

\author{
Ken Sahara \\ Division of Applied Biosciences \\ Graduate School of Agriculture \\ Hokkaido University \\ Sapporo 060-8589 \\ Japan
}

Tel \& FAX: ++81-11-706-2402

email: sahara@abs.agr.hokudai.ac.jp 
Abstract: Comparative genomic hybridization $(\mathrm{CGH})$ with a probe mixture of differently labeled genomic DNA from females and males highlighted the $\mathrm{W}$ chromosomes in mitotic plates and the $\mathrm{W}$ chromatin in polyploid interphase nuclei of the silkworm Bombyx mori, the flour moth Ephestia kuehniella, and the wax moth Galleria mellonella. The overproportionate fluorescence signal indicated an accumulation of repetitive sequences in the respective $\mathrm{W}$ chromosomes. Measurements of the fluorescence signals revealed two components, one that is present also in male DNA (non-W chromosomes) and another one that is present only in or preponderantly in female DNA (W chromosomes). While the $\mathrm{W}$ chromosomes of E. kuehniella and G. mellonella had both components, that of B. mori appeared to lack the latter component. Our results show that CGH can be applied to obtain a first estimate of the sequence composition of sex chromosomes in species from which otherwise little is known on the molecular level.

Key words: Lepidoptera, comparative genomic hybridization, molecular evolution, W chromosome. 


\section{Introduction}

Sex chromosomes very often stand out from the rest of the chromosomes by forming an unequal pair and by the partial or total absence of recombination. This applies to $\mathrm{X}$ and $\mathrm{Y}$ in male-heterogametic systems as well as to $\mathrm{W}$ and $\mathrm{Z}$ in female-heterogametic sex chromosome systems. The differences between $\mathrm{X}$ and $\mathrm{Y}$ or $\mathrm{W}$ and $\mathrm{Z}$ include size, centromere position, heterochromatin distribution, and sequence composition. Normally, the most conspicuous chromosome in a complement is the $\mathrm{Y}$ or $\mathrm{W}$ chromosome. The basis for this evolutionary trend is the constant heterozygosity of these chromosomes and their clonal transmission in the absence of meiotic recombination, conditions which allow an independent and rather uninhibited evolution.

Comparative genomic hybridization ( $\mathrm{CGH}$ ) had originally been developed to detect copy number differences between normal and cancer genomes on the cytogenetic level (Kallioniemi et al. 1992). When differentially labeled female and male genomes were used as probes, CGH identified molecularly differentiated sex chromosomes (Traut et al. 1999; 2001). Our goal was to use CGH to obtain an estimate for the extent of molecular differentiation of sex chromosomes. We applied the method to the $\mathrm{W}$ chromosomes of three species of Lepidoptera with unknown molecular composition of the sex chromosomes: the silkworm Bombyx mori, the flour moth Ephestia kuehniella, and the wax moth Galleria mellonella. The W chromosome forms a sex-specific body, the sex chromatin or W-chromatin, in female interphase nuclei (review: Traut and Marec 1996). We probed the $\mathrm{W}$ chromatin in polyploid nuclei rather than the $\mathrm{W}$ chromosomes in mitotic plates since the $\mathrm{W}$ chromatin of polyploid nuclei forms a single mass of considerable size and, in contrast to mitoses, sufficient numbers of such nuclei can easily be obtained for measurements.

\section{Materials and methods}

The silkworms, Bombyx mori (L.) (Bombycidae), were hybrids between Tw1 females and No males reared by K.S. (Sahara et al. 1990). Flour moth larvae, Ephestia kuehniella Zeller (Pyralidae), were from the wild-type strain 'L', kept at the Institut $\mathrm{f} r$ Biologie, L beck (Germany). Wax moth larvae, Galleria mellonella (L.) (Pyralidae), were from cultures of the Institute of Entomology, České Budějovice (Czech Republic).

Mitotic chromosomes were prepared from larval wing discs and ovaries. Polyploid nuclei were obtained from larval midgut cells. Pieces of midgut were shortly fixed in Carnoy fixative (ethanol, chloroform, acetic acid, 6:3:1), minced on a slide in a drop of $60 \%$ acetic acid and fixed to the slide using a heating plate at $50{ }^{\circ} \mathrm{C}$. Slides that contained a sufficient number of cytoplasm-free nuclei (checked with phase-contrast optics) were stored in the freezer until further use.

Whole genomic DNAs were isolated from larvae according to Blin and Stafford (1976). DNA was labeled by nick translation (Bionick Labeling system, Life Technologies, Karlsruhe, Germany) with FluorX-dCTP for DNA from females (f-probe) and Cy3-dCTP (Amersham Life 
Science, Arlinghton Heights, Illinois) for DNA from males (m-probe).

CGH was carried out according to Lapierre et al. (1998) with slight modifications. The probe cocktail for one slide ( $10 \mu 1,50 \%$ formamide, $10 \%$ dextran sulphate, 2 xSSC) contained $400 \mathrm{ng}$ labeled DNA from females, $400 \mathrm{ng}$ labeled DNA from males, $4 \mu \mathrm{g}$ unlabeled sonicated competitor DNA from males, and $25 \mu \mathrm{g}$ salmon sperm DNA. Hybridization was for three days at $37{ }^{\circ} \mathrm{C}$. A stringent wash at $62{ }^{\circ} \mathrm{C}$ in $0.1 \mathrm{xSSC}, 1 \%$ Triton $\mathrm{X}-100$, was followed by washes at room temperature, counterstaining in DAPI (4'6-diamidino-2-phenylindole) and mounting in antifade $(0.233 \mathrm{~g}$ 1,4-diazobicyclo(2.2.2)-octane in $1 \mathrm{ml} 0.2 \mathrm{M}$ Tris- $\mathrm{HCl}, \mathrm{pH} 8.0$ and $9 \mathrm{ml}$ glycerol).

Images were captured with a B\&W CCD camera separately for each fluorescent dye using the Zeiss Pinkel fluorescence filter set and superposed by means of the image processing program Adobe Photoshop Version 4.01. We used superposed B\&W images for quantitative analysis and pseudocoloured images for illustration (light blue for DAPI, green for f-probe, and red for m-probe). Average fluorescence intensities of the $\mathrm{W}$ chromatin body (W), the remaining chromatin (nonW) and the chromatin-free background (B) were measured in successive layers of the superposed image using the lasso tool and the histogram function of the Photoshop program package. The concentration (C) of f-probe (FluorX signals), m-probe (Cy3 signals) or DAPI bound to the $\mathrm{W}$ chromatin was calculated relative to the remaining chromatin according to the formula $\mathrm{C}=(\mathrm{W}$ B)/(nonW - B). The ratio of bound f-probe versus $\mathrm{m}$-probe in the $\mathrm{W}$ chromatin, therefore, compares with a 1:1 ratio in nonW chromatin. Theoretically, we should allow for a deviation from the 1:1 ratio because the $\mathrm{Z}$ chromosome is included in nonW chromatin but the error introduced by the $\mathrm{Z}$ chromosome is negligible as the diploid chromosomes numbers in the three species are high, $2 n=56$ in B. mori and $2 \mathrm{n}=60$ in E. kuehniella and G. mellonella.

\section{Results and Discussion}

In diploid mitotic complements of B. mori, E. kuehniella, and G. mellonella, CGH with differently labeled genomic DNAs from females (f-probe) and males (m-probe) highlighted a single element, the $\mathrm{W}$ chromosome (Figs. 1 - 3). The $\mathrm{W}$ in the three species was strongly labeled not only by the respective f-probe which contained the $\mathrm{W}$ chromosomal DNA but also by the m-probe which did not contain W chromosomal DNA. In contrast, the intensity of DAPI fluorescence was not noticeably higher than that of the remaining chromosomes. This indicated that the $\mathrm{W}$ chromosomes did not differ in DNA concentration but had accumulated faster annealing, repetitive sequences. Among them were, at least partly, DNA sequences that were common to both sexes, i.e. DNA sequences occurring also elsewhere in the genome.

For measurements of the bound probes and of DAPI fluorescence we used polyploid interphase nuclei of about 8 to $64 \mathrm{C}$ (Figs. 4 - 6). The concentration of DAPI fluorescence in the W chromatin was 1.5, 2.3, and 3.0 times higher (B. mori, G. mellonella, and E. kuehniella, respectively) 
than in the remaining chromatin (Table 1, 1st column). These figures reflect the denser packaging of the DNA in the heterochromatic $\mathrm{W}$ chromatin body relative to that in euchromatin. Concentrations of both, f-probe and m-probe, bound to the $\mathrm{W}$ chromatin were overproportionately higher, between 1.4 and 6.0 fold (m-probe/DAPI in E. kuehniella and f-probe/DAPI in G. mellonella, repectively) compared to the DAPI signals (Table 1, 2nd and 3rd column). This indicated an accumulation in the $\mathrm{W}$ chromatin of repetitive sequences (with faster hybridization kinetics, not completely suppressed by the unlabeled competitor DNA). The concentration of f-probe bound to the $\mathrm{W}$ chromatin was even higher than that of m-probe in G. mellonella (2.1 fold) and E. kuehniella (1.5 fold). In B. mori, however, f-probe and m-probe concentrations were about equal (Table 1, last column). These data identified two components of W chromosomal DNA: (1) repetitive DNA common to females and males, i.e. present also in autosomes and/or Z chromosomes, and (2) repetitive DNA exclusively or preponderantly present in females, i.e. DNA that is either W-specific or at least very highly concentrated in the $\mathrm{W}$. While the $\mathrm{W}$ chromosomes of all three species contained the first component, the second component was evident in W chromosomes of G. mellonella and E. kuehniella but not in B. mori. The data on B. mori are consistent with a previous approach to recover $\mathrm{W}$ chromosomal DNA fragments (Abe et al. 1998): the few that could be isolated contained transposons. Both types of components have already been identified in well studied Y chromosomes like those from the human, the mouse, or Drosophila (e.g. Kjellman et al. 1995). The results show that the CGH approach supplies first information on the extent and type of molecular differentiation of sex chromosomes, information that is helpful in species from which little or no other sequence information is yet available.

The accumulation of the two types of repetitive DNA in the W chromosome adds to the list of differences that have so far been observed in lepidopteran sex chromosomes: (i) size differences (e.g., Philosamia cynthia, Traut and Mosbacher 1968), (ii) number differences such as $\mathrm{W}_{1} \mathrm{~W}_{2} \mathrm{Z} / \mathrm{ZZ}$ (e.g., Phragmatobia fuliginosa, Seiler 1914) and $\mathrm{WZ}_{1} \mathrm{Z}_{2} / \mathrm{Z}_{1} \mathrm{Z}_{1} \mathrm{Z}_{2} \mathrm{Z}_{2}$ (e.g., Yponomeuta spp., Nilsson et al. 1988), which result from fission or fusion events, or Z/ZZ (e.g., Talaeporia tubulosa, Seiler 1921) resulting from total loss of the W, (iii) differences in pachytene chromomere pattern (e.g., Orgyia thyellina, Traut and Marec 1997), (iv) heterochromatinization of the W in interphase nuclei (e.g., Choristoneura fumiferana, Smith 1945), and (v) absence or loss of most gene functions in the W (e.g., Bombyx mori, Tazima 1964). All differentiation of the W chromosome must have evolved since this chromosome came into being at the basis of a phylogenetic branch that unites Ditrysia (Traut and Marec 1996) and Tischeriina (Lukhtanov 2000) and includes the great majority of lepidopteran species.

\section{Acknowledgements}

We thank Tom Tonka (České Budějovice, Czech Republic) for providing Galleria 
mellonella. K.S. received a grant from the Ministry of Education, Culture, Sports, Science and Technology of Japan. F.M. was supported by a Research Fellowship from the Alexander von Humboldt Foundation (Bonn, Germany) and by Grant No. A6007307 of the Grant Agency of the ASCR (Prague). 


\section{References}

Abe, H., Kanehara, M., Terada, T., Ohbayashi, F., Shimada, T., Kawai, S., Suzuki, M., Sugasaki, T., and Oshiki, T. 1998. Identification of novel random amplified polymorphic DNAs (RAPDs) on the $\mathrm{W}$ chromosome of the domesticated silkworm, Bombyx mori, and the wild silkworm, B. mandarina, and their retrotransposable element-related nucleotide sequences. Genes Genet. Syst. 73: 243-254.

Blin, N., and Stafford, D.W. 1976. A general method for isolation of high molecular weight DNAfrom eukaryotes. Nucleic Acids Res. 3: 2303-2308.

Kallioniemi, A., Kallioniemi, O.-P., Sudar, D., Rutovitz, D., Gray, J.W., Waldman, F., and Pinkel, D. 1992. Comparative genomic hybridization for molecular cytogenetic analysis of solid tumors. Science, 58: 818-821.

Kjellman, C., Sj gren, H.-O., and Widegren, B. 1995. The Y chromosome: A graveyard for endogenous retroviruses. Gene, 161: 163-170.

Lapierre, J.M., Cacheux, V., Da Silva, F., Collot, N., Hervy, N., Wiss, J., and Tachdjian, G. 1998. Comparative genomic hybridization: Technical development and cytogenetic aspects for routine use in clinical laboratories. Ann. Genet. (Paris), 41: 56-62

Lukhtanov, V.A. 2000. Sex chromatin and sex chromosome systems in nonditrysian Lepidoptera (Insecta). J. Zool. Sys. Evol. Res. 38: 73-79.

Nilsson, N.-O., L fstedt, C., and D vring, L. 1988. Unusual sex chromosome inheritance in six species of small ermine moths (Yponomeuta, Yponomeutidae, Lepidoptera). Hereditas, 108: 259-265.

Sahara, K., Kawamura, N., and Iizuka, T. 1990. Analysis of sex chromosome pairings in tetraploid females of the silkworm by using the sex-linked recessive gene (in Japanese, English abstract). J. Seric. Sci. Jpn. 59: 196-201.

Seiler, J. 1914. Das Verhalten der Geschlechtschromosomen bei Lepidopteren. Nebst einem Beitrag zur Kenntnis der Eireifung, Samenreifung und Befruchtung. Arch. Zellforsch. 13: 159-269.

Seiler, J. 1921. Geschlechtschromosomen-Untersuchung an Psychiden. II. Die Chromosomenzyklen von Fumea casta und Talaeporia tubulosa. Arch. Zellforsch. 16: 18-46.

Smith, S.G. 1945. Heteropycnosis as a means of diagnosing sex. J. Hered. 36: 195-196.

Tazima, Y. 1964. The Genetics of the Silkworm. Academic Press, London.

Traut, W., Eickhof, U., and Schorch, J.-C. 2001. Identification and analysis of sex chromosomes by comparative genomic hybridization (CGH). Methods Cell Sci. 23:157-163.

Traut, W., and Marec, F. 1996. Sex chromatin in Lepidoptera. Q. Rev. Biol. 71: 239-256.

Traut, W., and Marec, F. 1997. Sex chromosome differentiation in some species of Lepidoptera. Chromosome Res. 5: 283-291. 
Traut, W., and Mosbacher, G.C. 1968. Geschlechtschromatin bei Lepidopteren. Chromosoma, 25: 343-356.

Traut, W., Sahara, K., Otto, T.D., and Marec, F. 1999. Molecular differentiation of sex chromosomes probed by comparative genomic hybridization. Chromosoma, 108: 173-180. 


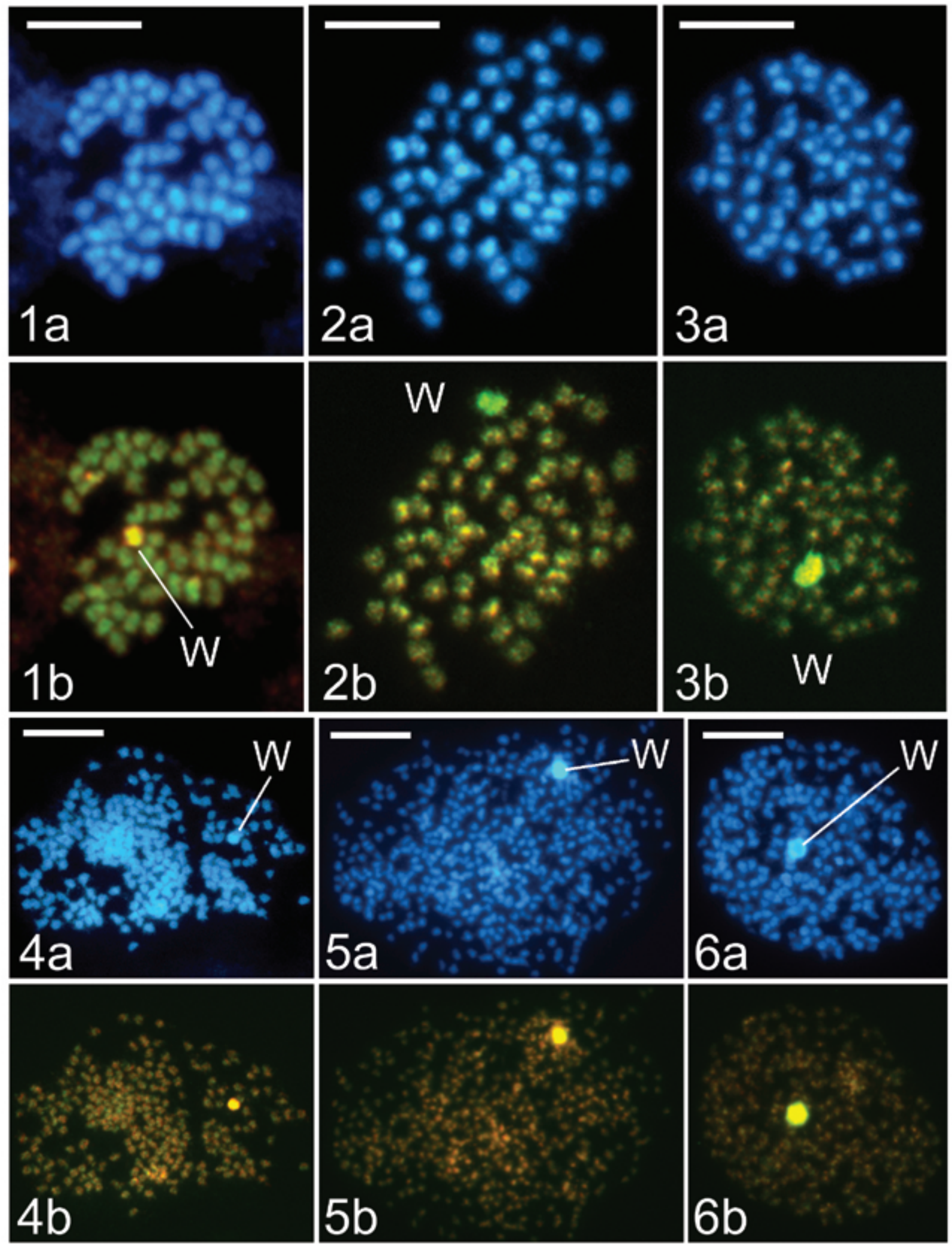

Sahara et al Fig. 1 


\section{Figure Legend}

Fig. 1 - 6. Mitotic metaphases (Fig. 1 - 3) and polyploid interphase nuclei (Fig. 4 - 6) of female larvae; note that interphase nuclei in Lepidoptera have a granular prophase-like texture. DAPI (a) and CGH (b) micrographs. CGH with FluorX-labeled DNA from females (green) and Cy3-labeled DNA from males (red). Figs. 1 and 4 Bombyx mori; Figs. 2 and 5 Ephestia kuehniella; Figs. 3 and 6 Galleria mellonella. W, W chromosome or W chromatin body. Scale bars $=10 \mu \mathrm{m}$. 
Table 1. Concentration of f-probe (DNA from females), m-probe (DNA from males), and DAPI bound to $\mathrm{W}$ chromatin bodies relative to non-W chromatin.

\begin{tabular}{lccccccccc}
\hline Species & $\mathrm{n}^{\mathrm{a}}$ & \multicolumn{2}{c}{$\mathrm{DAPI}^{\mathrm{b}}$} & \multicolumn{2}{c}{$\mathrm{f}$-probe } & \multicolumn{2}{c}{ m-probe } & \multicolumn{2}{c}{ f:m ratio } \\
\hline Bombyx mori & 24 & 1.50 & 0.37 & 4.76 & $1.62^{*}$ & 4.64 & 1.50 & 1.02 & $0.04^{\mathrm{d}}$ \\
Ephestia kuehniella & 20 & 3.03 & 0.67 & 6.63 & $2.13^{* *}$ & 4.33 & 1.01 & 1.52 & $0.20^{\mathrm{e}}$ \\
Galleria mellonella & 22 & 2.34 & 0.56 & 14.16 & $4.84^{* *}$ & 6.75 & 2.28 & 2.11 & $0.23^{\mathrm{f}}$ \\
\hline
\end{tabular}

a Number of polyploid nuclei measured.

$\mathrm{b}$ Means and standard deviation of $\mathrm{W}$ chromatin/non-W chromatin fluorescence ratio. *, the difference between $\mathrm{f}$-probe and $\mathrm{m}$-probe in Bombyx is marginally significant $(\mathrm{P}=0.015)$; **, the difference between $\mathrm{f}$-probe and $\mathrm{m}$-probe is highly significant $(\mathrm{P}<0.0001)$ (compared using paired two tailed t-test).

c The f-probe/m-probe ratio in $\mathrm{W}$ chromatin normalized for a 1:1 ratio in non-W chromatin; the differences between all three species were highly significant (letters $d, e$, or $f$ indicate statistical differences between groups within the column at $\mathrm{P}<0.001$; one way ANOVA with the Tukey-Kramer multiple comparison post test). 